\title{
Research on Strategic Human Resource Management of Enterprises Based on Cloud Computing
}

\section{Yue Hao}

College of Business Administration, Jilin Engineering Normal University, Changchun, China

18792044@qq.com

\section{Keywords: Cloud computing; Strategic human resource management; Human resources}

\begin{abstract}
Cloud computing technology can play a better supporting role in human resource management (HRM). The study shows that the characteristics of cloud computing technology and strategic HRM have internal consistency and the cloud computing technology can improve data utilization efficiency of HRM system, integrate management relationship, improve organizational structure, help enterprises to reduce the cost, improve efficiency and increase competitive advantages. The structure, technology and service function of strategic HRM system based on cloud computing are analyzed in this paper. At the same time, challenges that will be faced during the process of fusion between cloud computing and HRM system are also analyzed. In addition, countermeasures are put forward from perspectives of managers, enterprise management, organizational structure and risk resistance ability.
\end{abstract}

\section{Introduction}

According to strategic HRM, the appropriate human resources are vital strategic assets of enterprises. Every element of human resources should be analyzed, integrated and allocated depending on some rules and requirements. A set of relatively complete theories of management, institutes and methods will be built based on above results. Management of member's value and creative ability, integration of all the positions in human resources and support of strategic running aims of organizations are included in the set of theories of management, institutes and methods. These numerous and complex new content is a challenge to traditional HRM, so it is a necessary selection to achieve informatization of HRM by technological development.

Cloud computing, as a new generation of resource sharing utilization pattern, has characteristics of self-service and quantitative service[1]. It can increase utilization efficiency of human resource data to a large extent. Meanwhile, it can also integrate the chain between internal and external management and the chain between superior and low-level management, build a perfect organizational structure and play the advantage of fusion between its designing concept and human resource management. As a result, strategic HRM based on cloud computing broke from traditional modes, achieved off-site management and control by cloud management, decreased cost of HRM in enterprises and increased efficiency so that it can bring competitive advantages.

\section{Strategic HRM and Cloud Computing}

Cloud Computing and Its Characteristics. Strategic HRM is a challenge to traditional HRM mode. Traditional HRM can not deal with dynamic management mode, which is including numerous and complex content in constant variation. However, cloud computing can help to solve the problem. Cloud computing is a mode that is based on the increase, delivery and use of service related to the Internet. It is considered as a kind of virtual, dynamic and easy-expanded resources provided by the Internet [2]. There are three service levels in cloud computing: the infrastructure as the service, the platform as the service and the software as the service. The three service levels are achieved in infrastructure layer, software opening platform layer and application software layer. 
Characteristics of cloud computing are self-demanding service, dynamic resource configuration and quantitative service.

Effects of Cloud Computing on Enterprise Management. When cloud computing is applied in enterprises' management system, it can play its own special characteristics. It can help enterprises to decrease the cost of management, improve efficiency and get rid of limitations. The characteristics are as follows. The first one is the diversified selection. Management system based on cloud computing can appear in all the links of enterprise human resources. It stands for a kind of variation. It can also integrate the content of HRM and create the value. The second one is the decrease of the cost. If enterprises can use enterprises' management system with Saa S structure, they can decrease the cost of running and managing HRM tremendously. The third one is the increase of efficiency. Cloud computing has a mighty power in collecting and dealing with data information. It can also provide information management and procedure management, decision supporting, statistical analysis and other services so that enterprises can achieve automatic and electronic management. As a result, regular management of human resources will become more standard. The fourth one is to help enterprises get rid of limitations. Competitive position of enterprises, especially of micro, small and medium enterprises has been improved to a certain extent with the emergency of cloud computing. Micro, small and medium enterprises can enter the market fast and observe the variation of the market effectively by informational and technological services provided by public cloud. These enterprises can improve their competitiveness, get rid of limitations of enterprise scale and money. They can also use cloud computing as other large enterprises do.

\section{Strategic HRM System and Its Functions Based on Cloud Computing}

Strategic HRM System Based on Cloud Computing. It can be divided into two parts. The first one is the structure of strategic HRM system based on cloud computing. The Saa S system of cloud computing depends on SOA framework and Web Service [4]. The Saa S system can build some Web Service that is connected with HRM based on SOA framework. Hierarchical structure of strategic HRM system consists of these application modules and interface platform, network transferring database processing and other elements based on these application modules. The hierarchical structure is also called HRM cloud (As shown in figure 1).
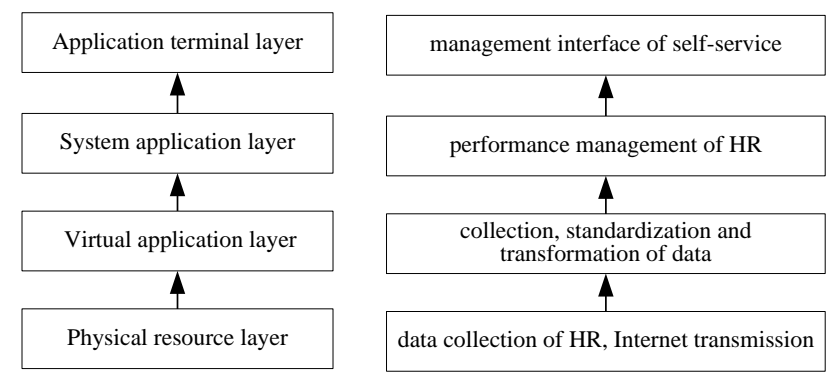

Figure 1. The hierarchical structure of strategic HRM system based on cloud computing

The second part is network protocol model of functional plate. The model consists of five modules. They are technology module, applied service module, storage module, formal module and application module.

The first one is the technology module. This module is composed of HRM technology, HRM process and maintenance of human resource cloud. The module provides running of the whole system with technological support, such as corresponding management technology, maintenance of cloud platform and so on.

The second one is the applied service module. This module is used to deal with cloud data and support good expression of data. 
The third one is the storage module. This module is composed of physical resource storage and virtual resource storage. Physical resource storage refers to physical storage that is done on the cloud platform of computers and it can transmit data to the upward layer. Virtual resource storage refers to website service data, accepting and processing of data and maintenance of cloud platforms.

The fourth one is the displaying module. It can display all kinds of service management interface so that users can access virtual resources by the displaying module.

The fifth one is the application module. Users can access corresponding resources by the browsers.

Strategic HRM System Function Based on Cloud Computing. This function has five modules. A more specific running methods of the management system can be constructed on the basis of protocol model of five functional modules. (As shown in figure 2), the functions of all the main components are stated in the below:

The first one is technological function of strategic HRM system based on cloud computing. It is also divided into four parts.

The first function is the recruitment management. The recruitment system based on cloud computing can support enterprises to publish the information and collect resumes through all the channels and deal with the resumes uniformly with a standard format that can be identified intelligently. It adopts redundant storage mode to make sure effectiveness and accuracy of every data. At the same time, it can avoid repeating inputs of data[4]. Enterprises can search and screen resumes at first. Enterprises also can learn quality, the ability and other index and data of applicants by Internet evaluation and give results back to applicants. After a few times of screening, enterprises can decide people who can be enrolled so that recruitment efficiency is improved as a whole[5].

The second function is the performance management. The performance management system based on cloud computing will pay more attention to standardization and systematization of performance assessing procedure. Cloud performance management system can analyze performance management aims scientifically and decompose strategic aims of enterprises into working aims that can be operated specifically. Every employee has different performance standards. According to characteristics of employees and characteristics of positions that are possessed by assessed objects, appropriate performance assessing tools need to be selected, such as KPI, 360-degree assessment and so on. Performance results of employees will be matched and contrasted with KPI automatically and then results are drawn.

The third function is the training management. The training management system based on cloud computing is included by a lot of knowledge and it can achieve real-time share and private ordering. Enterprises can achieve real-time share of resources by social Internet and building internal cloud knowledge base. Employees can acquire corresponding ability by sharing experience, skills and other knowledge mutually.

The fourth function is the wage management. The wage management system based on cloud computing, which adopts distributed storage, makes sure that the system can manage big data effectively. It has functions of digging and analyzing big data. Technology of cloud computing can make sure that wage computing of employees is more convenient and effective. 


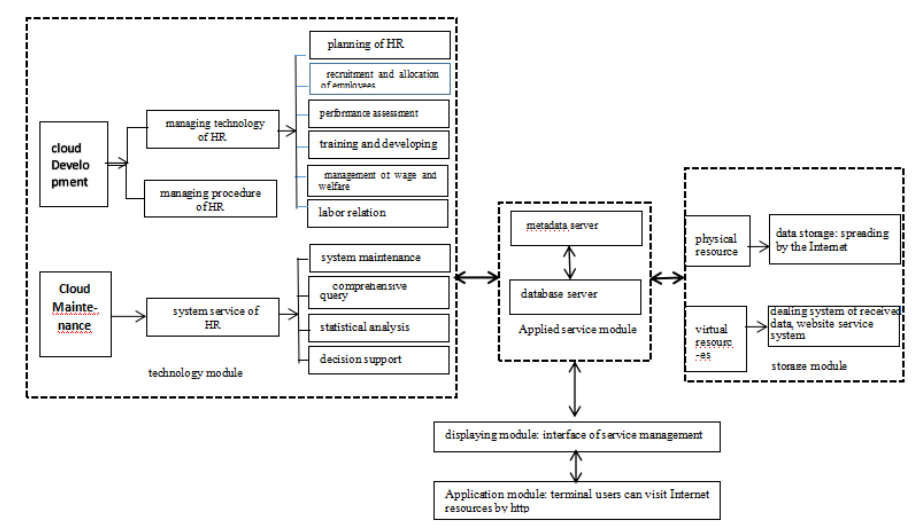

Figure 2. Strategic HRM system based on cloud computing

The second one is the service function of strategic HRM system based on cloud computing.

The first function is the data storage function. Cloud storage module can provide sharing database and solve traditional and relatively isolated problem about data source of HR. With the rapid development of enterprises, the mobility of people and cardinal number of people are both large. As a result, it is too difficult for enterprises to collect statistics of employees' number. However, cloud computing database can achieve real-time sharing of HR database among all the enterprises so that all the data can be connected effectively. Cloud computing database can save all the records on modifying and deleting data to make sure that data is complete and effective and improve management efficiency of HR. The second function is the synergy of applied function. Cloud management can enhance synergy between employees and enterprises. Employees can access HR database quickly and conveniently by cloud technology so that employees can gain information about welfare and wage. Signing in can be achieved at mobile terminals and the time sheets can be generated automatically so that enterprises can learn the state of employees. In addition, employees can connect with each other in time and collaborate smoothly in order to accomplish the work quickly.

\section{Challenge and Countermeasures of HRM in Enterprises Depending on Cloud Computing}

The combination of cloud computing and HRM can decrease the cost and increase competitive advantages. However, because of particularity of HRM and characteristics of cloud computing, the two are facing challenges during the fusion process. If managers do not pay attention to these challenges and can not deal with them, they may bring risks and bias for enterprises [8].

Challenges of Cloud Computing towards Human Resources Managers. The HRM system based on cloud computing can make office management transfer to procedure management. It is a challenge for human resources managers with low IT technology. Human resources managers should have more professional management knowledge, management tools and computer informational technology. Human resources managers should have the following ability in this cloud era: The first one is the ability of cloud strategy. Managers, as strategy executors, should not only provide enterprises with strategic supporting but also need to learn accurate positioning of enterprises and participate in the formulation of strategy of the enterprise. The second one is the ability of cloud coordination. HRM will be separated gradually because of expansion of enterprises' scale, so managers should play a coordinated role even if they are not authorized. They should take coordinated tasks, which include the relationship between the superior and the low-level, all the departments and newly enrolled employees. The third one is the ability of cloud learning. Managers should know that the learning point is the application and fusion of cloud technology in HRM. They need to become an expert in cloud management [9]. The fourth one is the ability of cloud consulting. It is necessary for managers to improve their own consulting ability so that they can provide tutoring and service of cloud management consulting, which is connected with enterprises' demand 
at any time. They should help enterprises to master the theory, methods and skills of cloud management. It means that they can become communicators who spread the cloud system smoothly in enterprises.

Challenges of Cloud Comuting towards Enterprise Management. The traditional HRM system still has some imperfect points in many aspects, such as position analysis and designing, wage management, performance management and informatization under the background of cloud computing application. Traditional HRM will not adapt to requirements in the new period of HRM. Aiming at different requirements, suppliers who do cloud service have developed an independent system with all the functions, such as recruitment, training and developing, performance assessment, wage and other system. Suppliers can provide outsourcing service for HRM in enterprises so that the new system can be more perfect than the original system. Enterprises can rent or buy independent functional systems according to their own situation, they can also formulate personalized innovative design, achieve managing reform and adapt to challenges of cloud computing based on the standardization of the cloud computing system [6].

Challenges of Cloud Computing towards the Organization and Structure of Enterprises. Cloud computing is not only a method to deal with information but also a method to integrate resources. In terms of the organization and structure of enterprises, the traditional hierarchical structure and the flat structure both can not adapt to new managing requirement under cloud computing mode. The market environment where enterprises are involved is more complex and variant and competition among them is stronger so that the original organization and structure play a gradually baffling role. The organization and structure of enterprises need to be transformed to a more flexible direction. It is also called cloud transformation of organization and structure. Enterprises can make the adjustment from two aspects. The first is that enterprises should adjust departmental responsibility and organization and structure according to strategic aims of enterprises, and then they should adjust position responsibility and re-allocate department responsibility. The second is that enterprises should make adjustment on aims of HRM and plans of all the positions.

Challenges of Cloud Computing towards the Ability of Risks Resistance in Enterprises. Cloud computing service involves sensitive information of individuals and enterprises. When incidents happen, the risks may appear. Firstly, an important characteristic of cloud computing service [9] is the lease by many users. It means that users can share the same resources. As a result, enterprises are facing risks of leaking confidential data because of resource sharing. If there is any loophole, data and a large number of information may be leaked under this situation. Secondly, management of cloud computing system belongs to service suppliers. Mergers, bankruptcy and other changes can happen. Data may be published. Service end and data may be transferred. Some changes maybe bring risks for enterprises. As a result, the safety problem of cloud computing is a challenge for enterprises. Enterprises must balance advantages and risks. Cloud computing suppliers can develop a more safe software system. Enterprises also can guarantee and strengthen cloud technology by contracts or agreements so that they can protect their own interests to the greatest degree.

\section{References}

[1] P.C.Du, M.Li and Y.Tong: Adaptive Transformation of Human Resource Management in the Cloud Computing Era [J], China Human Resources Development, 2013, (15). (In Chinese)

[2] W.L.Huang: Study on Application of Technology and Management in Small and Medium-Sized Enterprise Informationization In Cloud Computing[J], 2012, (6). (In Chinese)

[3] J.Zhu and J.W. Lai: A Review of the Research on Inter Organizational Information System Trust Based on Cloud Computing [J], Science and Technology Management Research, 2014, (11). (In Chinese)

[4] Y.M.Zhang and H.O.Liu: Cloud Computing Service Model and Strategy Driven by Collaborative Innovation [J], China Science and Technology Forum, 2013, (10) 1.(In Chinese) 
[5] J.He: Research on Enterprise Crisis Management System Based on Cloud Computing Technology under Large Data [J], Science and Technology Management Tesearch, 2014, (21). (In Chinese)

[6] Barrs $\mathrm{T}$ and Spruit M: Analysing the Security Risks of Cloud Adoption Using the SECA Model:A Case study [J], Jounal of Universal Computer Science,2012,18(12):1662-1678.

[7] Aleem A and Sprott C R: Let me in the cloud: Analysis of the Benefit and Risk Assessment of Cloud Platform[J], Journal of Financial Crime ,2013,20(1):6-24.

[8] Iyer B and Henderson J C: Preparing for the Future: Understanding the Seven Capabilities of Cloud Computing[J], MIS Quarterly Executive,2010,9(2):117-131.

[9] Zhang J and Seidmann A: Perpetual Versus Subscription Licensing under Quality Uncertainty and Network Externality Effects[J], Journal of Management Information Systems 2010,27(1):39-68.

[10] Aljabre: A.Cloud Computing for Increased Business Value[J], International Journal of Business and Social Science,2012,3(1):234-239. 